1

2

3

4

5

7

8

9

10

\title{
Simulated hail impacts on flexible photovoltaic laminates: testing and modelling
}

\author{
Mauro Corrado • Andrea Infuso · Marco Paggi
}

Received: date / Accepted: date

Abstract The problem of simulated low-velocity hail impacts on flexible photovoltaic (PV) modules resting on a substrate with variable stiffness is investigated. For this type of PV module it is shown that the prescriptions of the IEC 61215 International Standard for quality control used for rigid (glass-covered) PV modules should be augmented by taking into account the real mounting condition and the stiffness of the substrate in the simulated hail impact tests. Moreover, electroluminescence inspection of the crack pattern should be made in addition to electric power output measurements. An implicit finite element simulation of the contact problem in dynamics is also proposed, with two different degrees of accuracy, to interpret the experimentally observed extension of cracking. Results pinpoint the im-

M. Corrado, A. Infuso

Department of Structural, Geotechnical and Building Engineering, Politecnico di Torino

Corso Duca degli Abruzzi 24, 10129, Torino, Italy

Tel.: +39-011-0904858

Fax: +39-011-0904899

E-mail: mauro.corrado@polito.it

Present address: M. Corrado

Civil Engineering Institute, Materials Science and Engineering Institute, École Polytechnique Fédérale de Lausanne (EPFL), Station 18, CH-1015 Lausanne, Switzerland

M. Paggi

IMT School for Advanced Studies Lucca, Piazza San Francesco 19, 55100 Lucca, Italy portant role of stress wave propagation and reflection in the case of soft substrates.

Keywords Photovoltaic laminates · Low-velocity impacts · Experimental testing · Dynamic contact problem

\section{Introduction}

Photovoltaic (PV) modules are laminates composed of layers having very different mechanical properties and fulfilling different functions ranging from energy production to protection from the environment. Two main types are available on the market: $(i)$ rigid panels with a glass cover on the top of the stacking of layers to provide rigidity and protection against mechanical loads, typically installed in PV parks; (ii) semi-flexible panels to be bonded onto flat or curved substrates where protection is guaranteed by a polymeric layer which allows a certain degree of flexibility. Since PV modules are installed outdoor, thermo-hygrometric cycles, wind gusts, snow, and hail impacts are the main sources of damage and degradation during the expected working period of 25 years. The reader is referred to [1-6] for an overview of mechanical issues affecting the durability and the electric performance of PV modules. Among them, hail impacts are particularly severe for semi-flexible laminates, since the polymeric layers above the Silicon ( $\mathrm{Si}$ ) 
solar cells only slightly mitigate the effect of impacts. As a consequence, cracking in Silicon may occur, which can induce significant electrical power-losses.

Experimental tests simulating hail impacts were pioneeringly carried out in 1978 at the Jet Propulsion Laboratory of the California Institute of Technology [7]. Different types of PV modules were tested, differing mainly for the top layer material: tempered glass, annealed glass, acrylic sheet and Silicone potting. The impact tests were performed with a pneumatic gun, firing ice balls with different diameters, at different velocities. The results were analysed by means of visual inspections and through the determination of the electric power-loss from current-voltage (I-V) curves. The experiments pinpointed that no semi-flexible panels (those with a Silicone potting top layer) were capable of withstanding 1-inch diameter simulated hailstone impacting at a velocity of $23 \mathrm{~m} / \mathrm{s}$ or higher, without the occurrence of solar cell cracking. However, at that time it was not possible to analyse the experimental results by means of more sophisticated technologies, such as the electroluminescence (EL) technique, which permits nowadays to clearly identify the crack pattern and its effect on the electric response (the reader is referred to $[3,5,8$, 9] for the fundamentals of EL imaging and its working principles).

Today, in the European zone, all the various types of PV modules have to comply with the IEC 61215 standard [10], including also simulated hail impact tests to be performed by firing a molded ice ball with a pneumatic launcher onto the PV module. The standard diameter of the ball is $25 \mathrm{~mm}$, and the impact velocity is $23 \mathrm{~m} / \mathrm{s}$. However, different diameters and velocities can be used for special environments. The purpose of the test is to verify that the module is capable of withstanding the impact of hailstones. Hence, it provides a simple pass/fail assessment based on the visual inspection of the cover and the electric power-loss, rather than a quantitative evaluation of the amount of cracking in PV modules after impacts. Specifically, the following sequence of shots is requested to hit specific locations of the PV module, recording by visual inspection any sign of damage and visual effects:

1. A corner of the module window, not more than 50 $\mathrm{mm}$ from the frame (1 shot);

2. An edge of the module, not more than $12 \mathrm{~mm}$ from the frame (1 shot);

3. Over edges of cells, near an electrical joint ( 2 shots);

4. Over points of minimum spacing between cells (2 shots);

5. On the module window, not more than $12 \mathrm{~mm}$ from one of the points at which the module is fixed to the supporting structure (2 shots);

6. On the module window, at points farthest from the points selected above (2 shots);

7. Any points which may prove especially vulnerable to hail impact.

The requirements to pass the test are: $(i)$ absence of major visual defects; ( $i i)$ degradation of maximum output power less than $5 \%$ of the value measured before the test; ( iii) insulation resistance meeting the same requirements as for the initial measurements. However neither the type of substrate is mentioned in the testing procedure, nor the quantitative evaluation of the crack pattern morphology by electroluminescence is required. If this testing is suitable for rigid PV modules, where fracture of glass that can be visually assessed with the naked eye correlates well with the amount of cracking in Silicon, this is not the case of flexible PV modules. In this article we will show that the plastic cover usually remains undamaged after impacts, while Silicon cells exhibit cracking, whose extension does depend on the substrate stiffness. For modules resting onto stiff substrates, cracking tends to be concentrated near the impact point, giving rise to a small portion of electrically insulated area that can be detected by I-V curves, possibly leading to harmful hot spots. In the case of soft substrates, on the other hand, a much wider crack pattern takes place, but with most of the cracks still electrically conductive. As a consequence, they cannot be detected by a power measurement immediately after testing while, after moisture and ther- 
mal cycles inducing degradation, significant impact on power-losses can take place. Hence, for semi-flexible PV modules, it will be concluded that EL inspection should be performed and the type of substrate and mounting conditions should be included in the testing procedure, since they shall be essential parameters to judge about the suitability and safety of a flexible PV module depending on the specific application.

To shed light onto the effect of hail impacts on semiflexible PV modules from a quantitative point of view, a series of impact tests with a polyamide sphere fired with a pneumatic gun is proposed, reproducing the conditions for the simulation of hail impacts. The crack pattern is assessed by EL imaging and the main results are summarized in Section 2. To interpret the observed crack patterns and draw general conclusions, two different simulation strategies based on the finite element method and with different degrees of approximation are proposed in Section 3. Numerical trends in terms of expected extension of the crack pattern are compared with experimental results in Section 4.

The problem of impacts and contact mechanics in dynamics has been extensively investigated both from the computational methodology point of view [11-13] and the applications [14-18]. However, most of the contributions available in literature concern applications in the marine, aerospace and defence fields [16-20], where the commonly used composite sandwich panels are made up of two thin but stiff facesheets or skins separated by a lightweight and thick but low modulus core. Analogously, the problem of hail impacts has been mainly studied with reference to aerospace applications, characterized by high velocities [21-24]. On the contrary, the present problem involves composite laminates with soft polymeric encapsulant layers that have to be modeled as hyperelastic materials at finite strain, a layer of Silicon cells with a very brittle mechanical response, and the difficulty to assess the extension of cracking that cannot be made with the naked eye, all aspects not in common with composite shells for defence, marine and aerospace applications. The conclusions in Section
5 pinpoint that the crack pattern strongly depends on the impact velocity and on the stiffness of the substrate. Specific recommendations for the PV module installation and for acceptance depending on the application are finally devised.

\section{Experimental tests}

Commercial semi-flexible PV modules have been used for the experimental tests. They have a rectangular shape made of 2 rows of 5 monocrystalline Silicon solar cells each, see Fig. 1(a). Solar cells have a square shape with rounded corners and are connected with each other in series by two busbars, see Fig. 1(b). The size of the solar cells is $156 \times 156 \mathrm{~mm}^{2}$. These PV modules are made of five layers: a backsheet $0.345 \mathrm{~mm}$ thick, an epoxy-vinyl-acetate (EVA) layer with a thickness of $0.4 \mathrm{~mm}$, solar cells with a thickness of $0.166 \mathrm{~mm}$, another layer of EVA $0.6 \mathrm{~mm}$ thick, and a top protective layer in polyethylene terephthalate (PET) with a thickness of $0.265 \mathrm{~mm}$, see Fig. 1(c).

The impact of hailstone on PV modules was reproduced with low-velocity impact tests, performed by firing a polyamide sphere with a pneumatic gun. During the impact tests, the PV modules were laid on substrates with different mechanical properties in order to reproduce different realistic installation conditions and assess their role on cracking, an aspect not yet investigated in the literature and not taken into account in the IEC prescriptions. The effective extent of cracking created inside the solar cells after each impact of the sphere has been assessed by taking an EL image, which shows crack patterns and electrically inactive portions of the solar cell as dimmer areas.

[Fig. 1 about here.]

\subsection{Low-velocity impact tests}

Impact tests were carried out in the laboratory of Materials and Structures of the Department of Structural, Geotechnical and Building Engineering of Politecnico di 
Torino. A pneumatic gun built to perform low-velocity impact tests [25] (maximum velocity of $20 \mathrm{~m} / \mathrm{s}$ ) with a spherical projectile having a diameter of $40 \mathrm{~mm}$ was used (see Fig. 2). The pneumatic gun apparatus is composed by several parts, herein described in relation to Fig. 2:

1. An air compressor;

2. A tank for the storage of compressed air;

3. A vertical gun barrel. The spherical projectile is restrained at the top of it, with an electromagnetic jaw, manually unlocked by the operator;

4. A manual control tap to adjust the inlet air pressure;

5. A pressure gauge to read and control the air pressure stored in the storage tank;

6. A velocity gauge at the bottom end of the gun barrel to measure the output velocity of the projectile;

7. A rigid mounting plane, over which the PV module is placed.

A preliminary set of shots was performed in order to derive a calibration curve relating the pressure of the stored air to the velocity of the projectile measured at the exit of the gun barrel. The obtained curve is shown in Fig. 3(a). Moreover, this preliminary series of shots was used to select the appropriate velocity of the projectile to be used in the tests. IEC standards [10] prescribe to reproduce hailstone with molded ice balls, and provide a correlation between the impact velocity and the diameter of the sphere. However, this approach requires a careful examination of the ice balls before shooting, to avoid defective ice balls and ice cracks influencing the repeatability of the test itself.

For research purposes, to assure repeatability and propose also a much simpler testing procedure, a polyamide sphere with a diameter of $40 \mathrm{~mm}$ was used instead of ice balls. In this case, the prescriptions of the IEC standard cannot be used, and the conversion of the velocity in order to have the same linear momentum as for the ice balls cannot be applied. In fact, polyamide and ice have also a completely different material response during impact, namely linear elastic for polyamide and brittle for ice that shatters into many pieces, contribut- ing to energy dissipation. Hence, the selection of the velocity for the proposed impact tests was done in order to reproduce similar crack patterns and damage zones as in the case of ice. The crack patterns due to different impact velocities with polyamide spheres are shown in Fig. 3(b). Impacts with a velocity of 10 and $12 \mathrm{~m} / \mathrm{s}$ fully indent the solar cells with large dimmer areas representing electrically insulated regions, whereas impacts with a velocity of 6 and $7 \mathrm{~m} / \mathrm{s}$ produce a well identifiable crack pattern similar to the effects of ice ball impacts. In particular, the velocity of $6 \mathrm{~m} / \mathrm{s}$ was selected as the most appropriate for the present setup.

[Fig. 2 about here.]

[Fig. 3 about here.]

During tests, as it can be seen in the photo in Fig. 2, the PV module lies horizontally on the mounting plane, perpendicular to the gun, and as close as possible to the bottom end of the gun barrel in order to limit the effect of the gravity acceleration on the impact velocity and guarantee the reproducibility of the imposed impact velocity. Impact tests were performed by impacting a solar cell in its center to avoid spurious effects caused by busbars soldered on solar cells, as well as boundary effects.

Regarding the substrate interposed between the PV module and the rigid mounting plane, three cases were considered to simulate the conditions typical of different installation setups and analyze their effect on cracking. The three substrates, listed from the stiffer to the softer, are: (i) a wooden board; (ii) an alveolar polycarbonate (PC) layer interposed between the PV module and the wooden board; (iii) a polystyrene (EPS) layer interpotween the PV module and the wooden boar to as hard, medium and soft substrate, respectively.

\subsection{Analysis of the crack pattern by} electroluminescence imaging

Cracks and electrically inactive areas in Silicon solar cells are not usually visible with the naked eye. How- 
ever, they can be analyzed by means of EL imaging [3], which is a non-destructive technique based on the evaluation of the level of luminescence emitted by Silicon when subjected to an imposed electric potential in forward bias. Electrically insulated zones or cracks can be identified by their low EL intensity, thus resulting in dimmer areas. In the present tests, a voltage of $0.7 \mathrm{~V}$ was applied to the PV modules by the Genesys GENH60-12.5 (750W-1U, TDK Lambda) power supplier. EL emission was detected by the cooled digital 12 bit CCD camera pco.1300 solar, with a resolution of $1392 \times 1040$ pixel and equipped by the Schneider Kreuznach XNP F1.4 lens with SWIR coating 800-1800 nm. Tests were performed inside a dark room, shading all the possible sources of light to avoid reflection. Nearly the maximum aperture of the lens (F2) was selected, with an exposure time of $5 \mathrm{~s}$ to avoid heating of the PV module that can affect measurements. Post-processing of the acquired EL images was made by using the facilities of the software CamWare. In particular, cut-off filters of 600 and $8200 \mathrm{~nm}$ were used for all the images to make them comparable and remove very high and very low emission in the EL signal spectrum.

The EL images of the Si cells subjected to impact are shown in Fig. 4(d)-(f), for the three considered substrates whose sketch is shown above. For the case with hard substrate, damage remains localized in a small area around the impact point. Inside such a circular region, solar cells are heavily indented and electrically inactive, whereas outside it damage appears in the form of few radial cracks. The indentation point can lead to harmful hot spots. Two small black spots close to the impact point are due to the bouncing of the sphere after the main impact. The radius $r^{*}$ of the circular dimmer area is $7.5 \mathrm{~mm}$. By reducing the stiffness of the substrate to the medium case (see Fig. 4(e)), damage becomes less localized and it spreads over a wider region around the impact point. The crack pattern is characterized by both radial and circumferential cracks, the extension of the latter being larger than the former. The maximum radius $r^{*}$ of the circular area interested by the circumferential crack is $15.8 \mathrm{~mm}$. Finally, the crack pattern with the soft substrate is similar to that of the medium one, although with even more extended circumferential cracks (see Fig. $4(\mathrm{f})$ ). In this case, $r^{*}$ is equal to $31.0 \mathrm{~mm}$. On the contrary, the lengths of the radial cracks are almost the same as in the previous case.

[Fig. 4 about here.]

\subsection{Characterization of the substrate stiffness}

Besides the impact tests, indentation tests were carried out in order to quantify the stiffness of the substrates and characterize them by a specific parameter. This is also fundamental for the identification of the contact model parameters input for the numerical simulations. Hence, quasi-static indentation tests were carried out with a Zwick Allround electro-mechanical testing machine (maximum capacity of the load cell of $10 \mathrm{kN}$ ). The indenter had a cylindrical shape, with radius equal to $5 \mathrm{~mm}$ and length of $40 \mathrm{~mm}$. The test has been performed in the MUSAM-Lab at the IMT School for Advanced Studies Lucca for each of the three materials used as substrate, namely wood, alveolar PC, and EPS (see Fig. 5). The force vs. indentation curves for the three materials are shown in Fig. 6(a).

[Fig. 5 about here.]

[Fig. 6 about here.]

The contact stiffness $k_{\mathrm{sph}}$ of a sphere indenting a substrate can be deduced from the contact stiffness $k_{\text {cyl }}$ of a cylinder through basic contact mechanics formulae valid for a linear elastic continuum [26]. For a sphere, the indentation force $F_{\mathrm{sph}}$ is function of the indentation depth $u$ as:

$F_{\mathrm{sph}}=\frac{4}{3} E^{*} R_{\mathrm{sph}}^{1 / 2} u^{3 / 2}$

where $R_{\mathrm{sph}}$ is the radius of the indenting sphere and $E^{*}$ is an equivalent elastic modulus function of the elastic 
where $L$ is the length of the cylinder.

The area of contact in the two cases is also function of the indentation depth:

$a_{\mathrm{sph}}=\pi R_{\mathrm{sph}} u$

$a_{\text {cyl }}=L \sqrt{2 R_{\text {cyl }} u}$

The average contact pressure for the sphere can therefore be derived from Eqs. (1) and (3a):

$\bar{p}_{\mathrm{sph}}=\frac{F_{\mathrm{sph}}}{a_{\mathrm{sph}}}=\frac{4}{3 \pi} E^{*} \sqrt{\frac{u}{R_{\mathrm{sph}}}}$,

and from Eqs. (2) and (3b) for the cylinder:

$\bar{p}_{\mathrm{cyl}}=\frac{F_{\mathrm{cyl}}}{a_{\mathrm{cyl}}}=\frac{\pi}{8} E^{*} \sqrt{\frac{u}{2 R_{\mathrm{cyl}}}}$

The contact stiffness can be obtained as the derivative of the average pressure with respect to the indentation depth. For the sphere and the cylinder they read, respectively:

$k_{\mathrm{sph}}=\frac{\mathrm{d} \bar{p}_{\mathrm{sph}}}{\mathrm{d} u}=\frac{4}{6 \pi} \frac{E^{*}}{\sqrt{R_{\mathrm{sph}} u}}$

$k_{\mathrm{cyl}}=\frac{\mathrm{d} \bar{p}_{\mathrm{cyl}}}{\mathrm{d} u}=\frac{\pi}{8} \frac{E^{*}}{\sqrt{2 R_{\mathrm{cyl}} u}}$

Therefore, those relations allow the computation of the average stiffness due to the indentation of the halfplane by a sphere with radius $R_{\text {sph }}$ from the experimentally evaluated average stiffness of a cylinder with radius $R_{\text {cyl }}$ in contact with the same half-plane:

$k_{\mathrm{sph}}=\frac{16 \sqrt{2}}{3 \pi^{2}} \sqrt{\frac{R_{\mathrm{cyl}}}{R_{\mathrm{sph}}}} \kappa_{\mathrm{cyl}}$.

The contact stiffness of the three substrates subject to the indentation of a sphere with radius of $20 \mathrm{~mm}$ is computed as a function of the indentation depth from the curves shown in Fig. 6(a) and applying, in sequence, the formulae in Eqs. (5), (6b), and (7). The obtained curves are shown in Fig. 6(b). The vertical axis is in logarithmic scale to emphasize the different magnitude in the three cases. From the obtained curves, we notice that the contact stiffness depends on the indentation depth. As it will be shown in the next section, the maximum value of indentation reached during the tests and in the simulations differs from substrate to substrate and it increases by decreasing its stiffness. Therefore, based on the actual indentation depth experienced in the impact test experiments, the characteristic values to be used in input to the numerical simulations are marked by dashed lines in Fig. 6(b). In particular, they are $0.0065 \mathrm{~N} / \mathrm{mm}^{3}$ for EPS, $0.2 \mathrm{~N} / \mathrm{mm}^{3}$ for alveolar PC, and $5 \mathrm{~N} / \mathrm{mm}^{3}$ for wood. The ratio between the contact stiffness of the three cases with respect to EPS is $1: 30: 770$.

\section{Numerical modeling}

Two numerical approaches characterized by a different degree of approximation are herein proposed to simulate the contact problem of a sphere impacting on the PV module and assess the induced stress field, to interpret the crack patterns observed in the experiments.

The first approach is a simplified method in which a quasi-static nonlinear finite element contact problem is solved to predict the load vs. indentation response of the system. Afterwards, the equation describing the nonlinear single-degree-of-freedom (SDOF) spring-mass model is used to generalize the quasi-static solution to the dynamic regime.

The second approach is the most accurate as possible and it corresponds to the solution of the nonlinear dynamic contact problem by using a fully implicit finite element integration scheme in space and time. In both cases, the finite element analysis program FEAP [27] is used for the solution of the equation set resulting from the weak form of the problem and the imposition of the initial and boundary conditions representative of the experimental tests. The real problem is herein approximated by the axis-symmetric model shown in Fig. 7.

[Fig. 7 about here.] 
Linear quadrilateral finite elements are used to discretize the continuum. Linear elastic constitutive relations have been considered for all the materials composing the layers of the PV module and for the sphere, except for the epoxy material (EVA) encapsulating the solar cells. This is in fact a polymeric material with a pronounced thermo-viscoelastic behavior [28,29]. Due to the testing conditions of dynamic loading and constant room temperature, these two complex mechanical aspects can be neglected, and a neo-Hookean hyperelastic constitutive relation at room temperature has been adopted. The material parameters are listed in Tab. 1.

[Table 1 about here.]

An implicit time integration scheme based on the Newmark method, and in particular the constant average acceleration scheme, is used in dynamics. The contact problem is solved by using the node-to-segment contact strategy [13] to identify the finite elements in contact. The penalty method is adopted to impose the satisfaction of the unilateral contact constraint. In this regard, the penalty method was preferred over the method of Lagrange multipliers since it was possible to provide a physical meaning to the penalty stiffness to be used in the simulations. Different values of the penalty parameter have been considered along the contact interface between the PV module and the rigid mounting plane in order to simulate contact with substrates characterized by different indentation stiffness, to reproduce the conditions of the experimental tests. Specifically, the penalty parameters used for the contact problem along this interface are taken proportional to the values of the contact stiffness experimentally evaluated (see Section 2.3 ), i.e., $1 \times 10^{6} \mathrm{~N} / \mathrm{m}^{3}$ for the soft substrate, $3 \times 10^{7} \mathrm{~N} / \mathrm{m}^{3}$ for the medium substrate, and $7.7 \times 10^{8} \mathrm{~N} / \mathrm{m}^{3}$ for the hard substrate. Therefore, the ratio $1: 30: 770$ was preserved as in the experimental tests. Regarding the interface between the sphere and the laminate, a very high penalty parameter is set to avoid any spurious interpenetration of the sphere and satisfy almost exactly the unilateral contact problem along that plane.

\subsection{Nonlinear SDOF spring-mass model}

The problem of impact of a sphere on a PV module resting on a substrate can be approximated by a SDOF spring-mass model according to the methodology proposed in [30] (see Fig. 8(a)). Basically, the mass of the equivalent system is given by that of the sphere, since the mass of the portion of module interested by the impact is negligible. On the other hand, the stiffness of the equivalent spring has to take into account both the stiffness of the sphere and the contact stiffness. The governing equation for the SDOF system is:

$M \ddot{u}+K(u) u=0$

where $K(u)$ is the nonlinear stiffness of the spring, that can be determined from a quasi-static FE simulation of the sphere indenting the PV module. In particular, three different cases were examined, one for each substrate type. Simulations were performed under displacement control, i.e. the displacement of the boundary $\mathrm{AB}$ of the sphere (see Fig. 7) was increased step-bystep and the resulting force was computed as the sum of the reactions along the same boundary. The obtained quasi-static force vs. indentation depth curve is shown in Fig. 8(b) for the three substrates. Each curve was then approximated with a polynomial function of the 4th degree (the corresponding equations are also superimposed to Fig. 8(b)). The nonlinear stiffness entering Eq. (8) is therefore provided by the derivative of the polynomial functions fitting the force vs. indentation curves. Due to the nonlinear character of the problem, Eq. (8) was solved with a step-by-step numerical integration, assuming a constant acceleration within each time step, and imposing the initial conditions of indentation $u(t=0)=0$ and velocity $\dot{u}(t=0)=6 \mathrm{~m} / \mathrm{s}$.

[Fig. 8 about here.] 
3.2 Rigorous implicit dynamic finite element solution

The alternative approach to simulate the impact tests is based on the finite element method applied to the dynamic problem with a fully implicit time stepping technique. The solution strategy adopted for this case is a step-by-step implicit dynamics, using the Newmark constant average acceleration scheme for time integration. At each time step, the Newton-Raphson incrementaliterative scheme is adopted to solve the nonlinear set of algebraic equations. The mass matrix of the finite elements was computed in lumped form and energy dissipation phenomena, for instance due to friction, were neglected. Due to the hyperelastic behavior of the EVA material, and in order to avoid instabilities, the time step was changed during the simulation, starting from $1 \mu \mathrm{s}$ and decreasing it down to $0.1 \mu \mathrm{s}$ by approaching the maximum indentation depth. As discussed in the Section 4, the present approach is the most accurate as possible, and it will be shown that it is more precise than the SDOF spring-mass model for the analysis of the stress field. On the other hand, it is computationally much more expensive, since it has to deal with dynamics, contact mechanics, and the nonlinear hyperelasticity of EVA at the same time.

\section{Results}

In this section, the predictions of the two numerical methods used to simulate the impact test are reported. Furthermore, they are compared with the experimental results in order to assess the possibility of using numerical simulations to predict the extension of the area subject to cracking caused by impacts. First, the analysis of the results is carried out in term of global response, i.e., by examining the relation between indentation depth and time. Then, a more detailed analysis is carried out by inspecting the stress field inside the PV module, with particular focus on the layer containing the solar cells.

\subsection{Indentation vs. time response}

The outcome of the numerical simulations, in terms of indentation depth vs. time and velocity vs. time, is shown in Fig. 9. The dashed lines refer to the solution obtained by a quasi-static finite element simulation combined by the SDOF spring-mass model to extend the solution to dynamics, whereas the solid lines refer to the finite element prediction by solving the contact problem in dynamics. In both cases, the simulations are stopped at the maximum indentation. Clearly, the two approaches provided almost identical results. As expected, the maximum indentation and the duration of the impact do depend on the substrate stiffness. In particular, a maximum indentation of $1.16 \mathrm{~mm}$ is achieved after $0.26 \mathrm{~ms}$ for the hard substrate, $2.20 \mathrm{~mm}$ after $0.54 \mathrm{~ms}$ for the medium substrate, and $4.54 \mathrm{~mm}$ after $1.14 \mathrm{~ms}$ for the soft substrate. Correspondingly, the negative gradient of the velocity increases by augmenting the substrate stiffness.

[Fig. 9 about here.]

\subsection{Analysis of the stress field}

The stress field inside the PV module is herein examined in order to verify whether it is possible to correlate the stress distributions in the Silicon layer to the extension of the cracked area observed in the EL images. Considering the brittle behavior of Silicon, it is reasonable to assume that cracking takes place as soon as the radial stresses reach the ultimate tensile strength of the material. Hence, even by assuming linear elasticity for Silicon, it is reasonable to expect that all the portion of the solar cell where the radial stress overcomes the ultimate tensile strength of Silicon correlates with the extension of the electrically inactive zones. Moreover, the point where the circumferential stresses become lower than such a threshold could be used to identify the maximum length of the radial cracks observed in the experiments. 
Due to the axis-symmetry of the model, the stress field is identified through the radial stress, $\sigma_{r}$, and the circumferential stress, $\sigma_{\theta}$. In the case of the SDOF springmass model, the stress field is quantified from a quasistatic simulation up to a deformation level corresponding to a maximum indentation depth predicted by the SDOF method. As an example, the contour plots of the radial stresses are shown in Fig. 10 for the three considered substrates. The highest stress level is located inside the solar cell, which has a Young modulus from 2 to 4 orders of magnitude higher than that of the other materials composing the PV laminate. The indentation of the sphere in the module determines a localized flexural state in the impacted solar cell. Therefore, the maximum and minimum stresses are achieved on the lower and upper surfaces of the layers. From a qualitative comparison of the three contour plots, the region subjected to the highest stresses increases by decreasing the substrate stiffness, in qualitative agreement with the experimental evidences.

[Fig. 10 about here.]

In order to quantitatively compare the three cases, the distributions of the radial and circumferential stresses along the radial coordinate at the bottom surface of the solar cell, i.e., the surface further from the side of impact, are shown in Figs. 11 and 12. In the SDOF spring-mass model, since there are no dynamic effects explicitly taken into account, the condition of maximum indentation corresponds to the achievement of the maximum radial stress in the module (see Fig. 11) and this simplifies the estimation of the extension of the cracked area of Silicon.

On the other hand, the same procedure is not viable for the rigorous finite element solution of the dynamic problem. The identification of the maximum and minimum stresses at the boundary of the solar cells is in fact complicated by the phenomenon of stress wave propagation which is not accounted for in the SDOF method. As a consequence of this, the peak stress in the various points along the boundary of the solar cells is reached at different time steps. Therefore, in this case, an envelope diagram of the stresses is required to assess the maximum extension of the solar cell layer experiencing a radial stress higher than the Silicon ultimate tensile strength. Hence, the diagram in Fig. 12 represents, for each material point, the maximum and the minimum radial stresses reached during the entire load history in the dynamic simulation.

The radial and circumferential stress distributions shown in Fig. 11 for the SDOF method and the corresponding envelope diagrams obtained from finite element dynamic simulations shown in Fig. 12 display common features. The maximum values of the radial and circumferential stresses take place within the area of contact between the ball and the PV module. The maximum radial stress $\sigma_{r}$ is found in a point about $3 \mathrm{~mm}$ far from the symmetry line. Further from the impact area, the radial stresses change sign, and the minimum values take place at a distance from 6 to $12 \mathrm{~mm}$ from the symmetry line. After the minimum, the radial stresses tend to vanish at a distance sufficiently far from the point of impact. The circumferential stresses $\sigma_{\theta}$ also tend to zero far from the point of impact, but without changing sign.

[Fig. 11 about here.]

[Fig. 12 about here.]

The diagrams in Figs. 11 and 12 are now interpreted in relation to the EL images showing dimmer areas (see Fig. 4(b)) in order to assess the possibility of using these numerical predictions to identify the region of the solar cell that becomes electrically inactive. As mentioned before, the key idea is to define such a region as the area where the absolute value of the radial stresses $\sigma_{r}$ overcome a threshold given by the ultimate tensile strength of Silicon. The use of the absolute value is justified by the fact that, on the upper surface of the solar cell, stresses are almost the same as those acting on the bottom surface and shown in Figs. 11 and 12, but with opposite sign due to bending. To this purpose, 
the envelope diagrams obtained from dynamic finite element simulations are considered first.

Considering a threshold value of $60 \mathrm{MPa}$ for $\sigma_{r}$ (see the horizontal dashed lines in Fig. 12), the region potentially subjected to circumferential cracks is predicted to have an extension defined by a radius $r^{*}$ of $10.5 \mathrm{~mm}$ for the hard substrate, $17.8 \mathrm{~mm}$ for the medium one, and $31.3 \mathrm{~mm}$ for the soft substrate. These values are in very good agreement with the experimentally observed extension of the crack patterns (see Fig. 4). The same threshold applied to the diagrams of the circumferential stress $\sigma_{\theta}$ identifies regions where radial cracks can develop with a radius of $6.8 \mathrm{~mm}$ for the hard substrate, $10.2 \mathrm{~mm}$ for the medium one, and $16.5 \mathrm{~mm}$ for the soft substrate. Based on these results, the extension of the solar cell portion subjected to radial cracks is predicted to be smaller than that subjected to circumferential cracks, which is fully consistent with the experimental observation (see Fig. 4).

The same analysis of the stress diagrams resulting from the SDOF method (see Fig. 11) does not lead to the same level of agreement with the experimental observation of the extension of the crack pattern, especially for the soft substrate. This is particularly evident for the radial stresses. In fact, for the same threshold of $60 \mathrm{MPa}$, SDOF predictions for $r^{*}$ are $9.4 \mathrm{~mm}$ for the hard substrate, $14.2 \mathrm{~mm}$ for the medium one, and only $20.3 \mathrm{~mm}$ for the soft substrate. A direct comparison between experimental results and numerical predictions based on the two proposed models for the extension of the cracked area, $r^{*}$, is provided in Fig. 13. Predictions by finite element simulations in dynamics are in excellent agreement with experimental results, since they lie very close to the dashed line at $45^{\circ}$. The SDOF method should be used for hard or medium stiff substrates.

[Fig. 13 about here.]

It is worth noting that the tensile strength of intact mono-crystalline silicon is usually much higher than the threshold value assumed in this analysis. However, solar cells used to produce PV modules are cut from a big cylindrical wafer and are subject to several industrial processes up to their final incapsulation in the EVA layers. All of these steps can induce impurities, microcracks and defects leading to stress concentrations and a much lower nominal tensile strength.

\section{Discussion and conclusions}

The impact of simulated hailstone on semi-flexible PV modules has been studied from the experimental and the numerical points of view. With respect to [7], the use of the EL technique represents a significant step forward for the accurate detection and analysis of the crack pattern. The use of EL imaging for a quantitative analysis of electric damage and crack pattern is highly recommended in the case of semi-flexible PV modules that are much more vulnerable to hail impacts than the rigid ones. Moreover, the effect of the type of substrate was also found to be very important and should be considered in the design of PV installations for the assessment of potential safety risks connected to hot spots induced by cracking.

As far as the numerical modeling is concerned, the proposed simplified approach based on quasi-static finite element simulations and the application of the SDOF model provides excellent results in terms of global response, i.e., indentation depth vs. time behavior. However, stresses predicted by this approach to be used to predict the extension of the crack pattern do not satisfactorily correlate with the experimentally observed dimmer areas in the EL images, especially for soft substrates. On the other hand, fully implicit finite element simulations in dynamics accounting for stress wave propagation and reflection provide stress diagrams that can be effectively used to assess the extension of cracking in solar cells due to impacts, for any substrate stiffness. In particular, the use of a nominal ultimate tensile strength of $60 \mathrm{MPa}$ to identify the extension of the crack zones leads to predictions that compare very well with experimental observations. Therefore, the proposed method can be used in practical engineering applications to as- 
sess different laminate configurations both in terms of layers thickness and material composition, and in relation to different mounting substrates.

As regards future perspectives, the analysis of the evolution of the crack patterns induced by impacts due to the exposure to cyclic thermal loads, performed by means of experimental tests inside a climate chamber, will be of high relevance for a complete evaluation of the actual durability of cracked PV modules. In fact, a further propagation of cracks and an increase of their opening can lead to an expansion of the electrically inactive solar cell areas, with a consequent increase of power-loss [6]. In this regard, the crack pattern with partial electric insulation observed for the soft substrate (see Fig. 4(b)), almost impossible to be assessed from the inspection of $\mathrm{I}-\mathrm{V}$ curves, could be quite dangerous in perspective, since it can lead to a very large electrically insulated area and a hot spot after a series of cyclic thermal loading in the field.

Acknowledgements This research has received funding from the European Research Council under the European Union's Seventh Framework Programme (FP/2007-2013)/ERC Grant Agreement No. 306622 (ERC Starting Grant "Multi-field and multi-scale Computational Approach to Design and Durability of PhotoVoltaic Modules" - CA2PVM).

\section{References}

1. Meyer EL, van Dyk EE (2004) Assessing the reliability and degradation of photovoltaic module performance parameters. IEEE Trans on Reliability 53:83-92.

2. Paggi M, Kajari-Schröder S, Eitner U (2011) Thermomechanical deformations in photovoltaic laminates. J Strain Analysis for Engineering Design 46:772-782

3. Paggi M, Berardone I, Infuso A, Corrado M (2014) Fatigue degradation and electric recovery in Silicon solar cells embedded in photovoltaic modules. Sci Rep 4:4506.

4. Paggi M, Corrado M, Rodriguez MA (2013) A multiphysics and multi-scale numerical approach to microcracking and power-loss in photovoltaic modules. Comp Struct 95:630-638.

5. Paggi M, Berardone I, Corrado M (2016) A global/local approach for the prediction of the electric response of cracked solar cells in photovoltaic modules under the ac- tion of mechanical loads. Engineering Fracture Mechanics, in press. doi:10.1016/j.engfracmech.2016.01.018

6. Lenarda P, Paggi M (2016) A geometrical multi-scale numerical method for coupled hygro-thermo-mechanical problems in photovoltaic laminates. Comput Mech, in press, doi:10.1007/s00466-016-1271-5.

7. Moore D, Wilson A, Ross R (1978) Simulated hail impact testing of photovoltaic solar panels. Prooc 24th Annual Technical Meeting, Institute of Environmental Sciences, Ft. Worth, TX, April 18-20, 1978, 419-430.

8. Sapora A, Paggi M, (2013) A coupled cohesive zone model for transient analysis of thermoelastic interface debonding. Comput Mech 53:845-857.

9. Berardone I, Corrado M, Paggi M (2014) A generalized electric model for mono and polycrystalline silicon in the presence of cracks and random defects. Energy Procedia $55: 22-29$.

10. International Standards IEC 61215:2006 (2006) Crystalline Silicon Terrestrial Photovoltaic (PV) Modules - Design Qualification and Type Approval.

11. Wriggers P, Vu Van T, Stein E (1990) Finite element formulation of large deformation impact-contact problems with friction. Comput Struct 37:319-331.

12. Laursen TA (2002) Computational Contact and Impact Mechanics. Springer-Verlag, Berlin, Germany.

13. Wriggers P (2006) Computational Contact Mechanics. Springer-Verlag, Berlin, Germany.

14. Yu C, Ortiz M, Rosakis AJ (2003) 3D modelling of impact failure in sandwich structures. European Structural Integrity Society 32:527-537.

15. Zavarise G, Bacchetto A (2006) Nail shooting on a steel support. In: Practical Applications Using Computational Contact Mechanics (Prez-Aparicio, JL ed.), The TCN Series on Simulation Based Engineering and Sciences, Vol. 2. Consorzio TCN, Trento, Italy, pp. 143-196.

16. Richardson MOW, Wisheart MJ (1996) Review of lowvelocity impact properties of composite materials. Composites: Part A 27A:1123-1131.

17. Xu LR, Rosakis AJ (2002) Impact failure characteristics in sandwich structures Part I: Basic failure mode selection. Int J Solids Struct 39:4215-4235.

18. Xu LR, Rosakis AJ (2002) Impact failure characteristics in sandwich structures Part II: Effects of impact speed and interfacial strength. Int J Solids Struct 39:4237-4248.

19. Akil Hazizan Md, Cantwell WJ (2002) The low velocity impact response of foam-based sandwich structures. Composites: Part B 33:193-204.

20. Ivañez I, Sanchez-Saez S (2013) Numerical modelling of the low-velocity impact response of composite sandwich beams with honeycomb core. Comp Struct 106:716-723. 
21. Kim H, Kedward KT (2000) Modeling hail ice impacts and predicting impact damage initiation in composite structures. AIAA J 38:1278-1288.

22. Kim H, Welch DA, Kedward KT (2003) Experimental investigation of high velocity ice impacts on woven carbon/epoxy composite panels. Composites: Part A 34:25-41.

23. Anghileri M, Castelletti L-ML, Invernizzi F, Mascheroni M (2005) A survey of numerical models for hail impact analysis using explicit finite element codes. Int J Impact Engng 31:929-944.

24. Olsson R, Juntikka R, Asp LE (2013) High velocity hail impact on composite laminates - Modelling and testing. In: Dynamic Failure of Composite and Sandwich Structures. Abrate S, Castani B, Rajapakse, YDS (Eds), Springer, pp. 393-426.

25. Nelva R, Morra L (2009) L'effetto della grandine su cupole e lucernari. ZENITAL, 1-49.

26. Johnson KL (1985) Contact Mechanics. Cambridge University Press, Cambridge, UK.

27. Zienkiewicz OC, Taylor RL (2000) The Finite Element Method. 5th ed., Butterworth-Heinemann, Oxford, UK.

28. Paggi M, Sapora A (2015) An accurate thermoviscoelastic rheological model for Ethylene Vinyl Acetate based on fractional calculus. Int J Photoenergy, paper No. 252740.

29. Ojo SO, Sapora A (2016) A thermo-visco-elastic shear-lag model for the prediction of residual stresses in photovoltaic modules after lamination. Comp Struct 136:481-492.

30. Abrate S (2001) Modeling of impacts on composite structures. Comp Struct 51:129-138. 


\section{List of Figures}

1 Semi-flexible PV module: (a) photo of one of the tested modules; (b) detail of a solar cell; (c) sketch of the cross-section of the PV module with its different layers. . . . . . . . . . . . . . . . . . . . . 14

2 Equipment for the impact test: (a) sketch; (b) picture of the pneumatic gun. . . . . . . . . . . . . . 15

3 (a) Calibration curve relating air pressure and output velocity of the projectile; (b) electrically damaged areas for different impact velocities. . . . . . . . . . . . . . . . . . . . . . . 16

4 Experimentally simulated hail impact tests: (a)-(c) sketches of the three considered substrates; (d)-(f) EL images showing the resulting crack patterns and electrically inactive areas. . . . . . . . . . . . 17

5 Photos of the indentation tests on: (a) wooden board; (b) alveolar polycarbonate; (c) polystyrene. . . 18

6 Results of the experimental indentation tests: (a) force vs. indentation depth and (b) average inden-

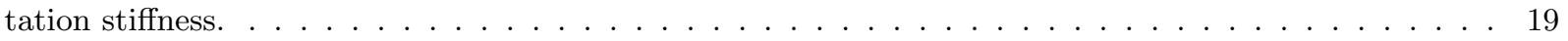

7 Sketch of the axis-symmetric model geometry for FE simulations. . . . . . . . . . . . . . . . . . . . 20

8 (a) Sketch of the SDOF spring-mass model; (b) indentation force vs. indentation depth curves from quasi-static finite element simulations, for the three considered substrates. . . . . . . . . . . . . . . 21

9 Results of the numerical simulations for different substrate stiffnesses: (a) indentation depth vs. time; (b) velocity vs. time. The dashed lines refer to the SDOF spring-mass model whereas the solid lines to the rigorous finite element solution in dynamics. . . . . . . . . . . . . . . . . . . . . . 22

10 Contour plot of the radial stress in the PV module for the three considered substrates. These predictions are obtained based on the SDOF method. . . . . . . . . . . . . . . . . . . . . 23

11 Stress distributions along the radial coordinate at the bottom surface of the Si cell predicted by the SDOF method: (a) radial stress; (b) circumferential stress. . . . . . . . . . . . . . . . . . . . . . . . . . 24

12 Envelopes of the stresses along the radial coordinate at the bottom surface of the Si cell predicted by dynamic finite element simulations: (a) radial stress; (b) circumferential stress. . . . . . . . . . . . . 25

13 Comparison between experimental results and numerical predictions of the extension of the silicon area cracked by circumferential cracks, $r^{*} \ldots \ldots \ldots \ldots$ 
1

2

3

4

5

6

7

8

9

10

11

12

13

14

15

16

17

18

19

20

21

22

23

24

25

26

27

28

29

30

31

32

33

34

35

36

37

38

39

40

41

42

43

44

45

46

47

48

49

50

51

52

53

54

55

56

57

58

59

60

61

62

63

64

65
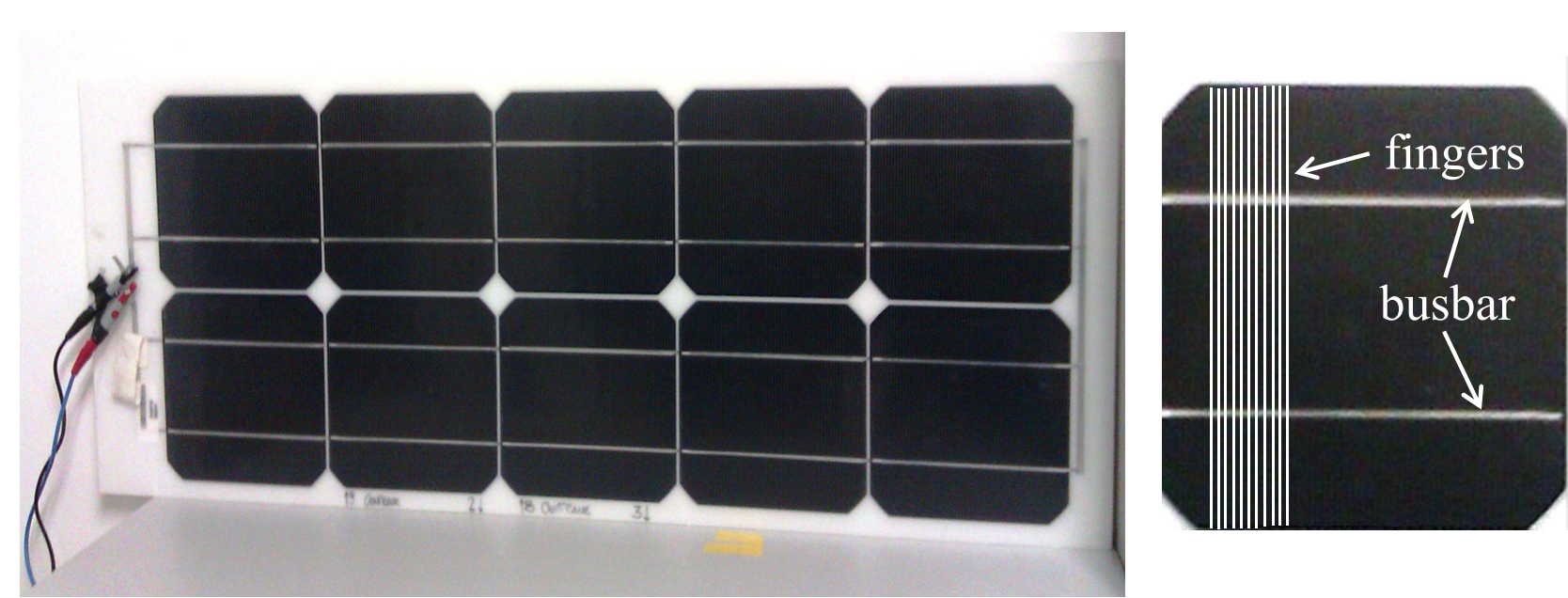

(a)

(b)

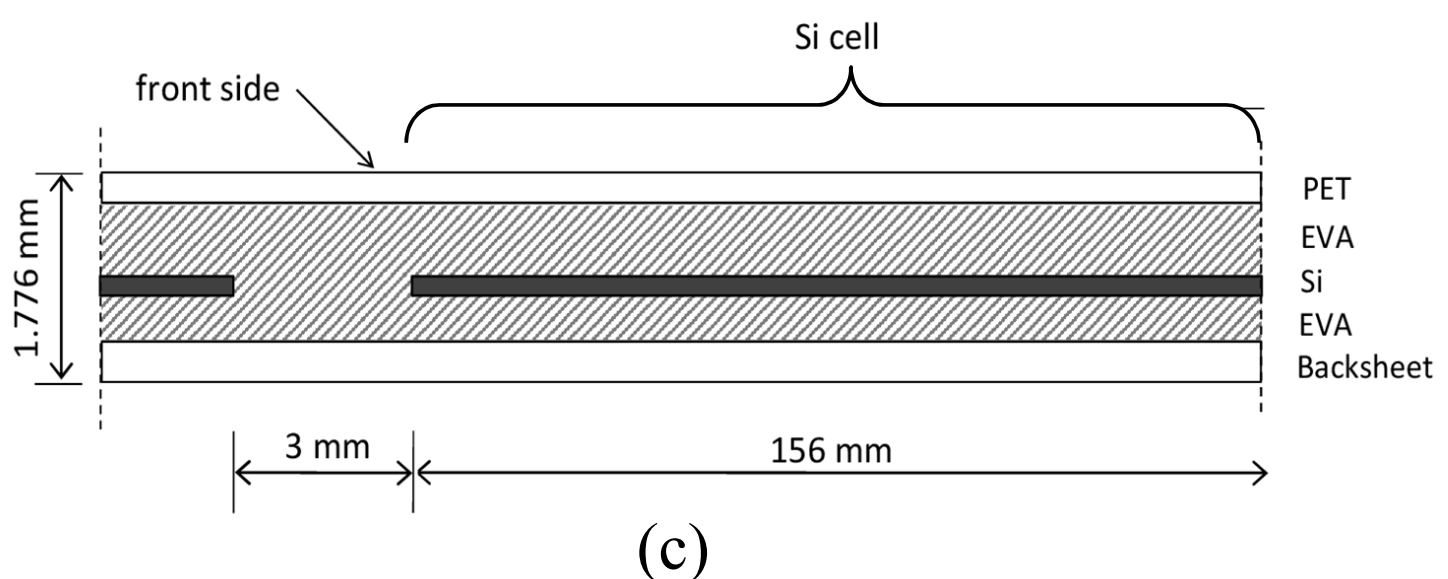

Fig. 1 Semi-flexible PV module: (a) photo of one of the tested modules; (b) detail of a solar cell; (c) sketch of the cross-section of the PV module with its different layers. 
1

2

3

4

5

6

7

8

9

10

11

12

13

14

15

16

17

18

19

20

21

22

23

24

25

26

27

28

29

30

31

32

33

34

35

36

37

38

39

40

41

42

43

44

45

46

47

48

49

50

51

52

53

54

55

56

57

58

59

60

61

62

63

64

65

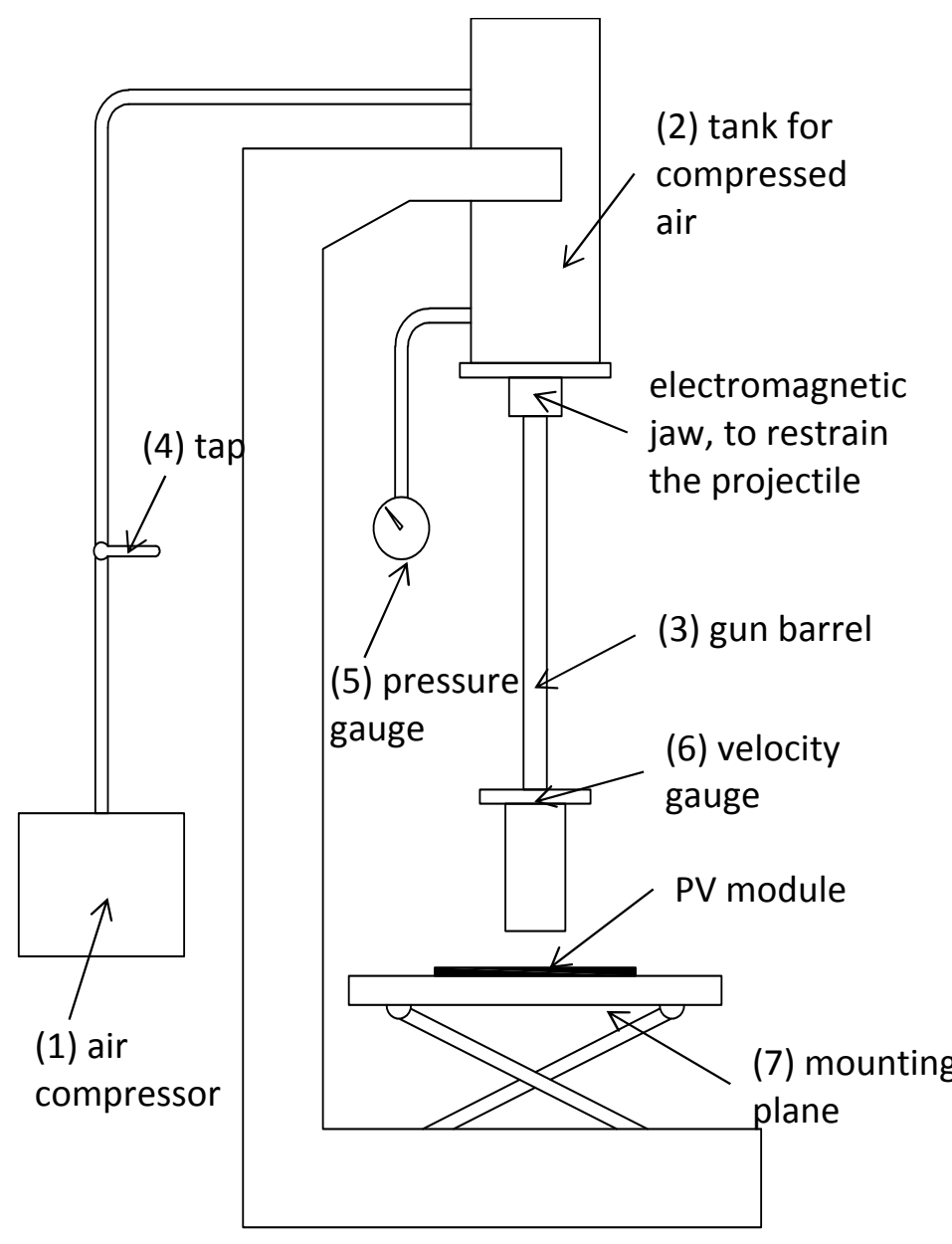

(a)

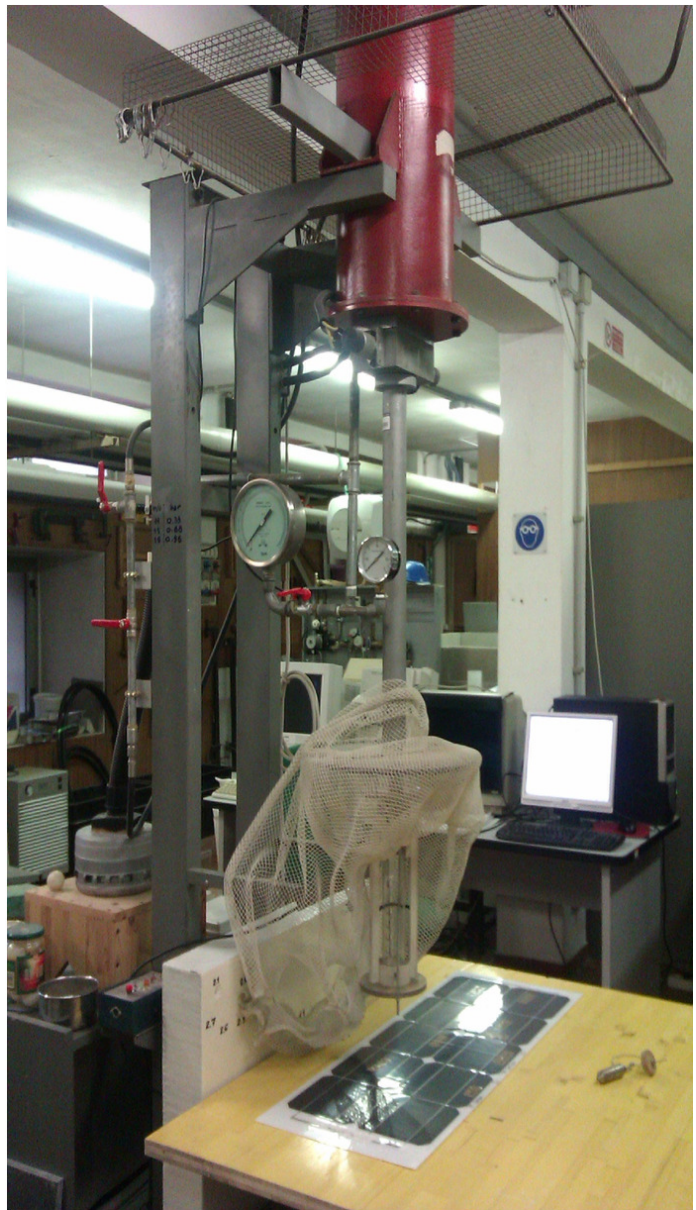

(b)

Fig. 2 Equipment for the impact test: (a) sketch; (b) picture of the pneumatic gun. 
1

2

3

4

5

6

7

8

10

11

12

13

14

15

16

17

18

19

20

21

22

23

24

25

26

27

28

29

30

31

32

33

34

35

36

37

38

39

40

41

42

43

44

45

46

47

48

49

50

51

52

53

54

55

56

57

58

59

60

61

62

63

64

65

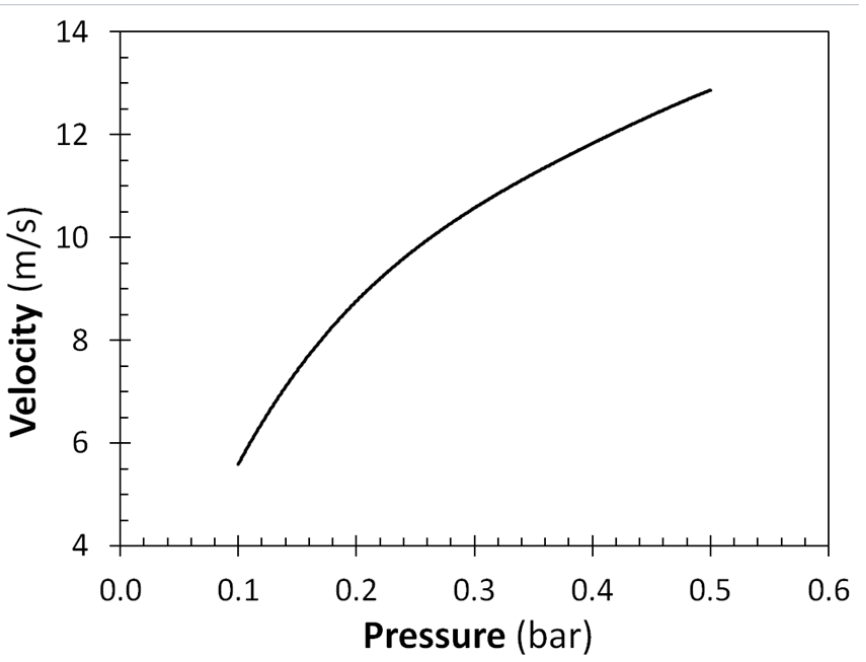

(a)

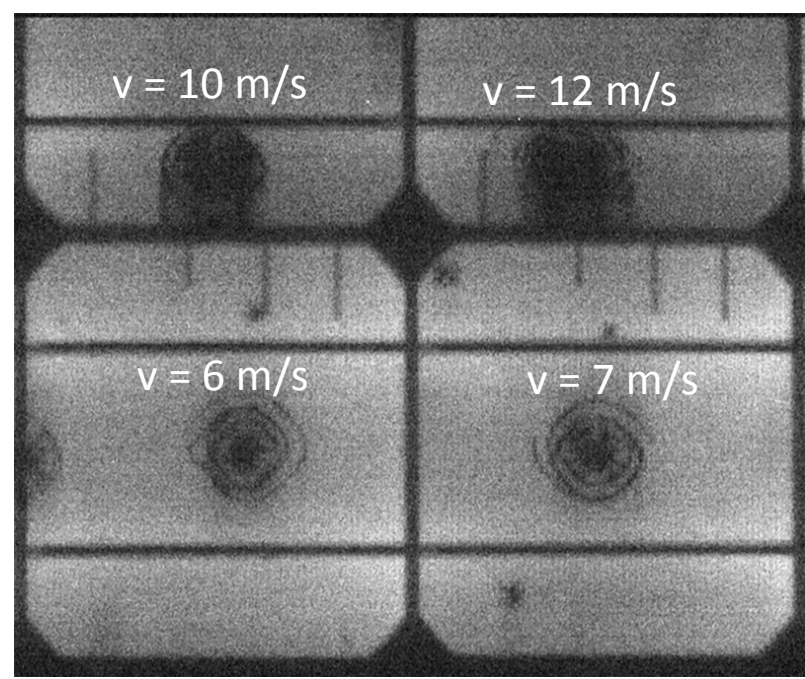

(b)

Fig. 3 (a) Calibration curve relating air pressure and output velocity of the projectile; (b) electrically damaged areas for different impact velocities. 


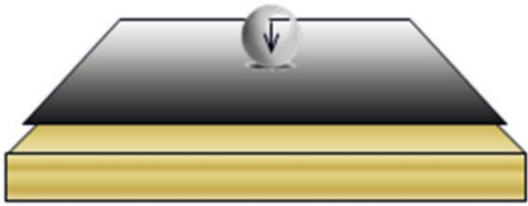

(a) Hard substrate: wooden board

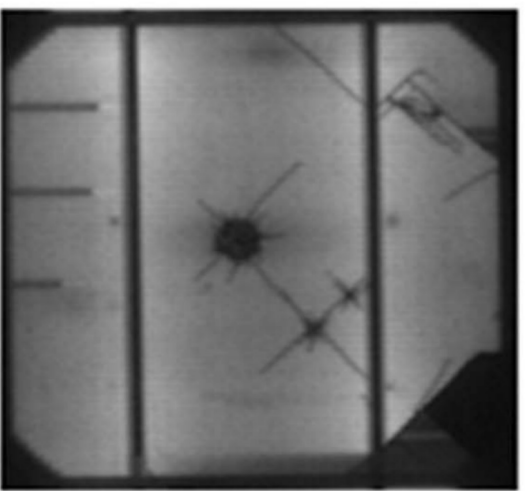

(d) Hard substrate

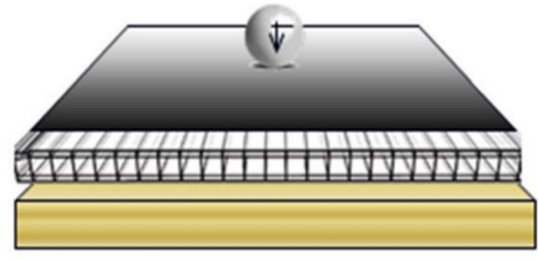

(b) Medium substrate: alveolar PC + wooden board

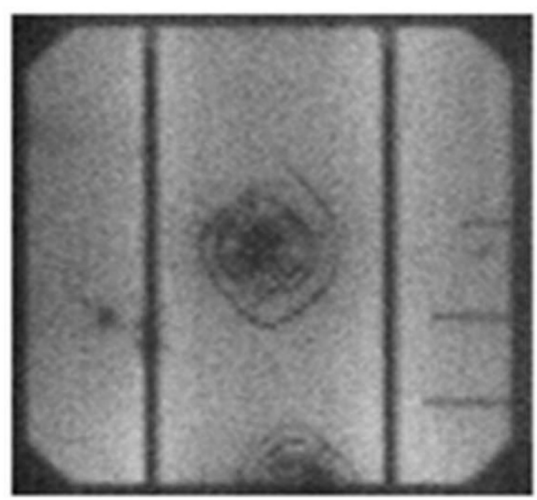

(e) Medium substrate

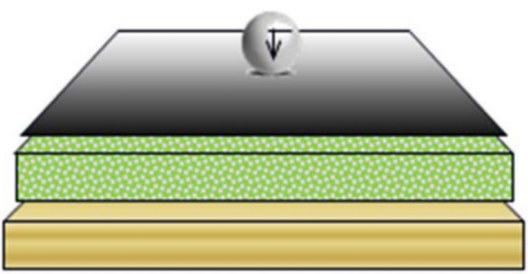

(c) Soft substrate: polystyrene + wooden board

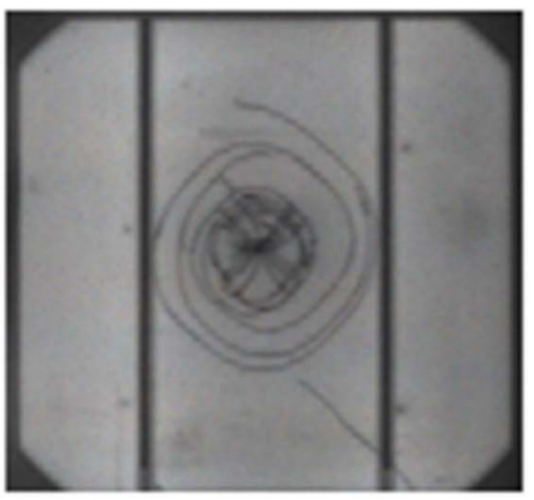

(f) Soft substrate

Fig. 4 Experimentally simulated hail impact tests: (a)-(c) sketches of the three considered substrates; (d)-(f) EL images showing the resulting crack patterns and electrically inactive areas. 


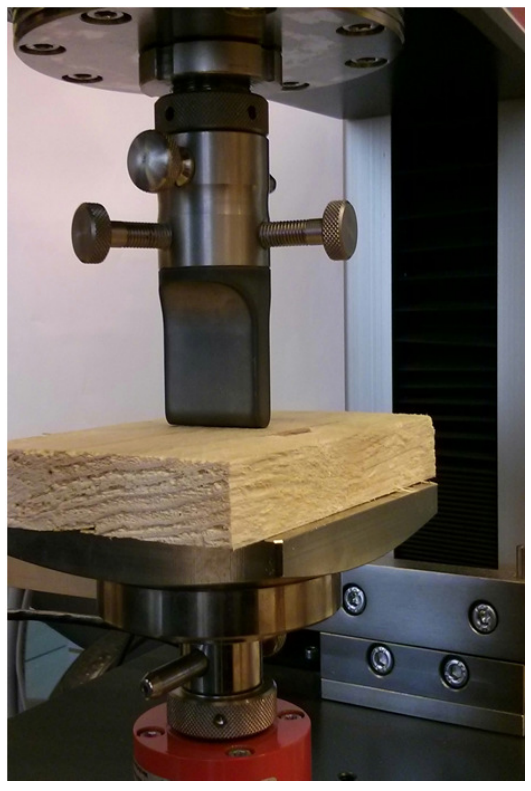

(a)

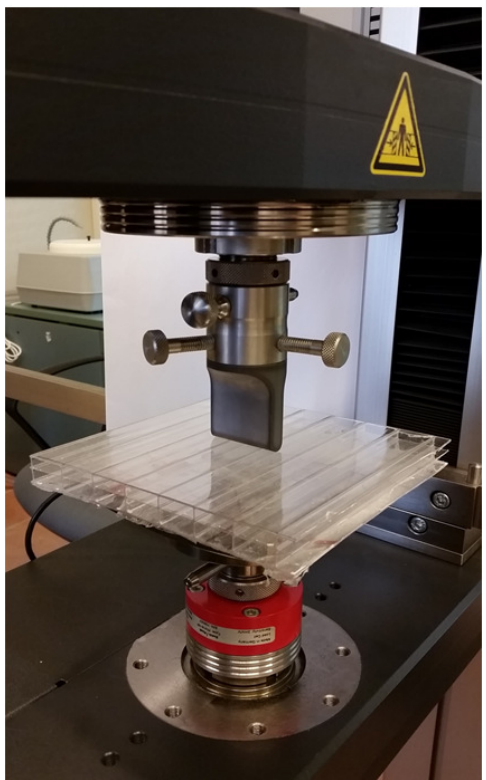

(b)

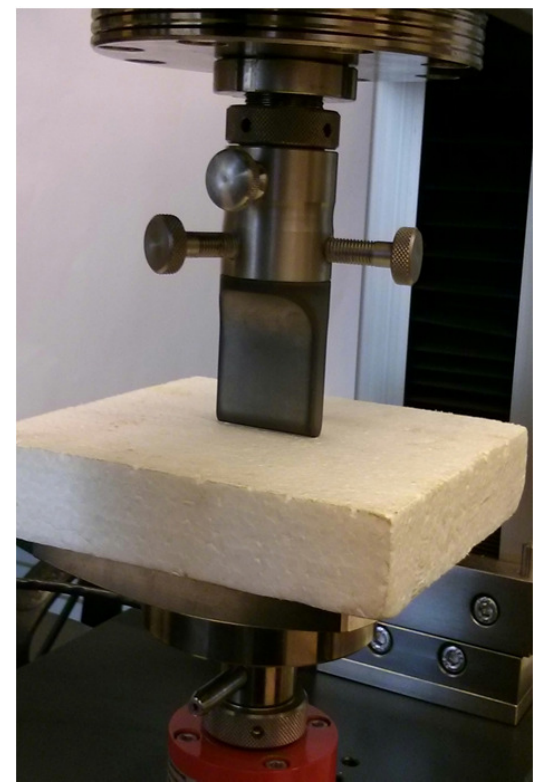

(c)

Fig. 5 Photos of the indentation tests on: (a) wooden board; (b) alveolar polycarbonate; (c) polystyrene. 


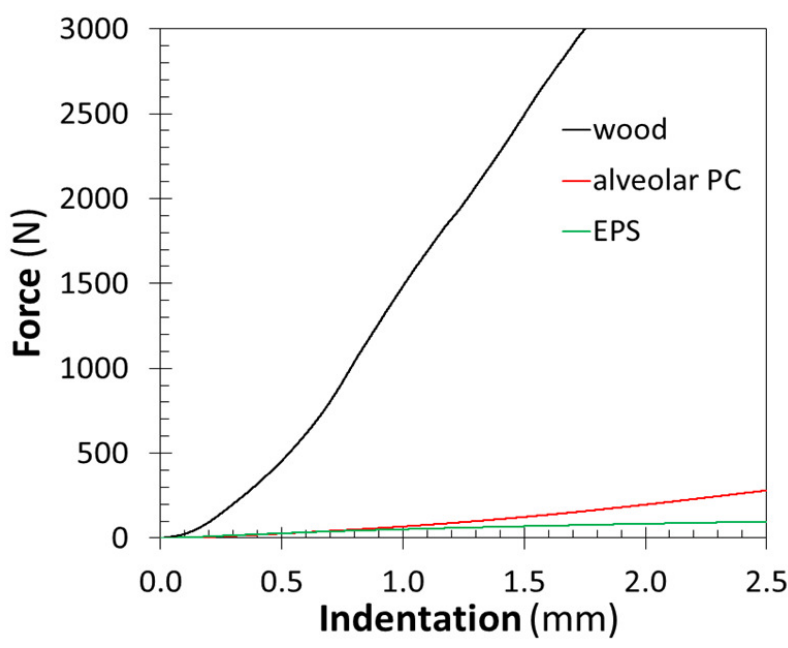

(a)

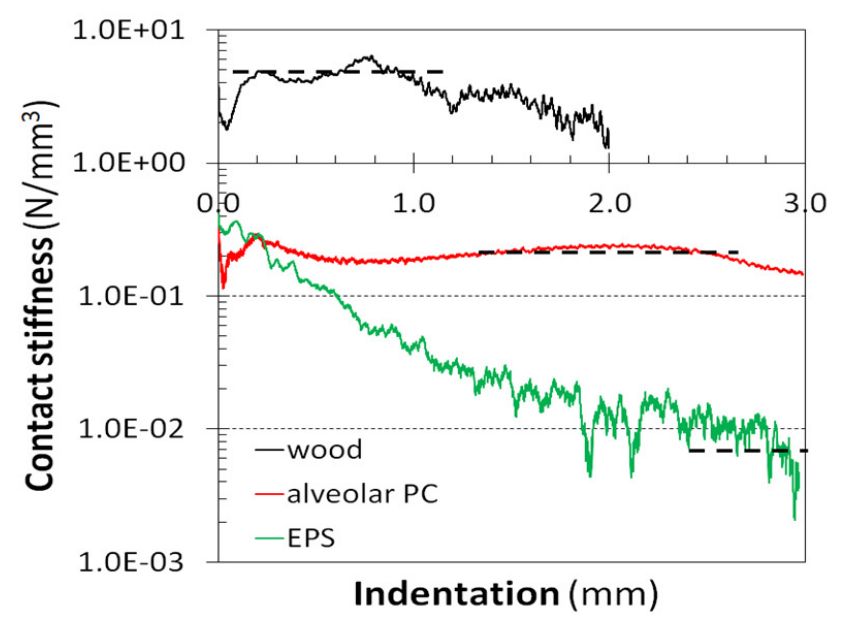

(b)

Fig. 6 Results of the experimental indentation tests: (a) force vs. indentation depth and (b) average indentation stiffness. 
1

2

3 approach for contact

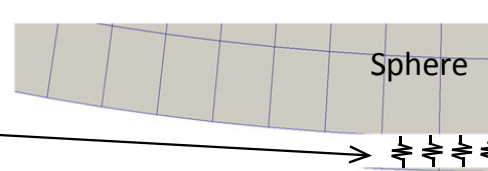

\section{PET}

EVA

penalty parameter approach to model

the substrate
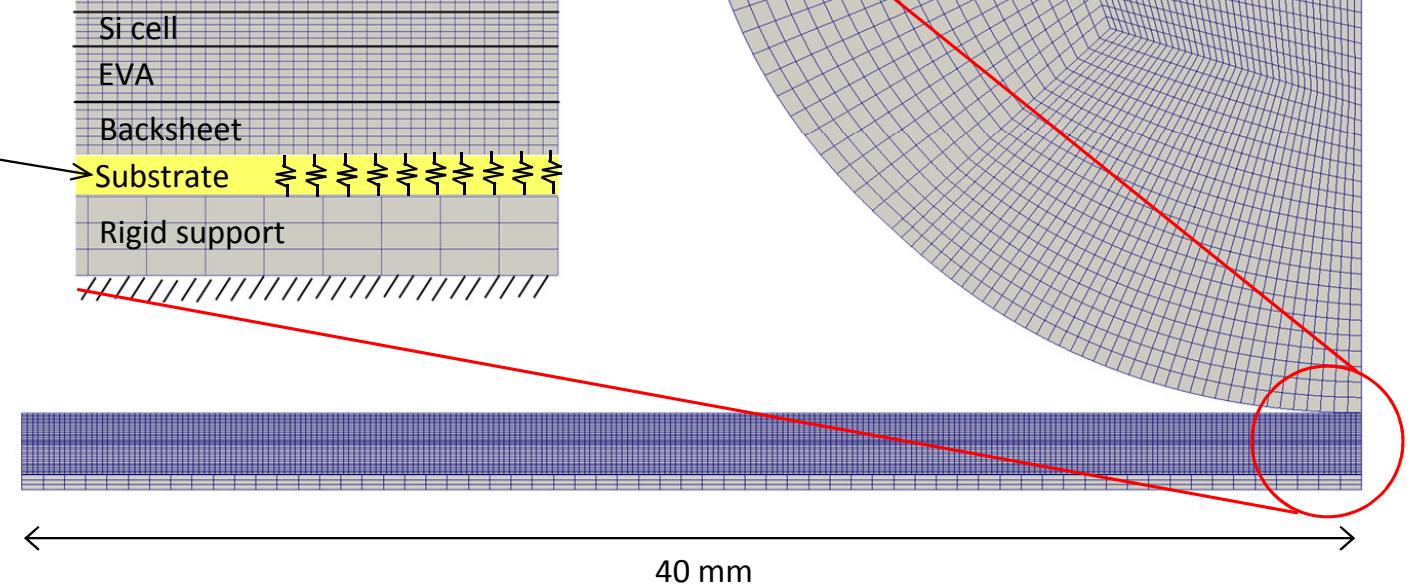

$40 \mathrm{~mm}$

Fig. 7 Sketch of the axis-symmetric model geometry for FE simulations.
$20 \mathrm{~mm}$

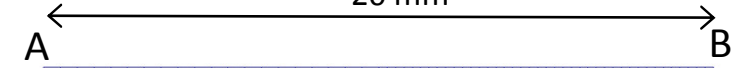

$\mathrm{B}$ 


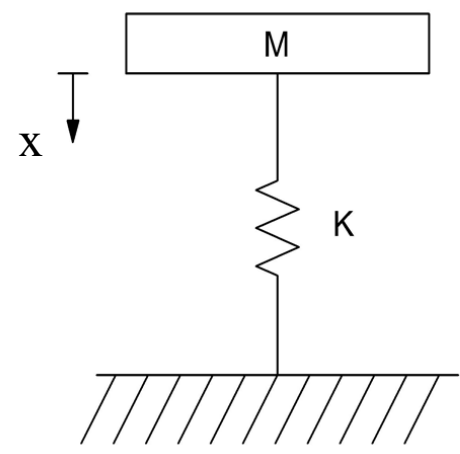

(a)

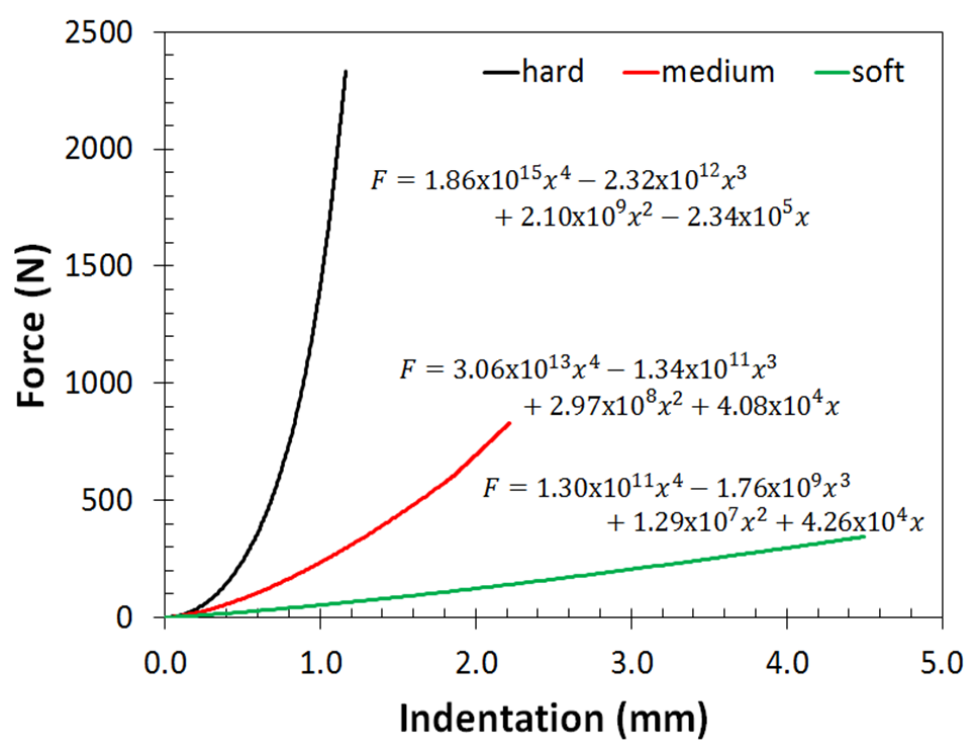

(b)

Fig. 8 (a) Sketch of the SDOF spring-mass model; (b) indentation force vs. indentation depth curves from quasi-static finite element simulations, for the three considered substrates. 
1

2

3

4

5

6

7

8

9

10

11

12

13

14

15

16

17

18

19

20

21

22

23

24

25

26

27

28

29

30

31

32

33

34

35

36

37

38

39

40

41

42

43

44

45

46

47

48

49

50

51

52

53

54

55

56

57

58

59

60

61

62

63

64

65

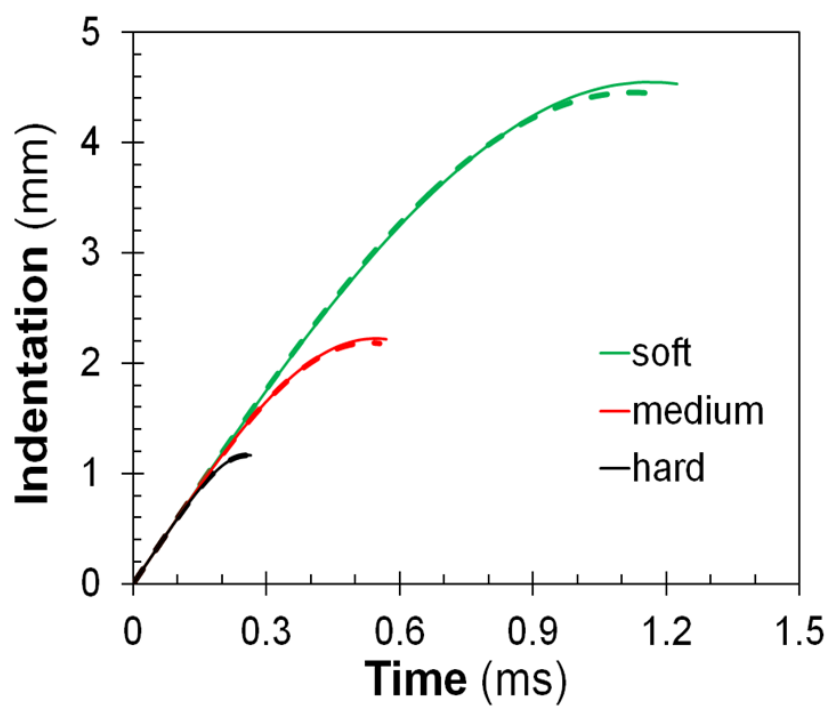

(a)

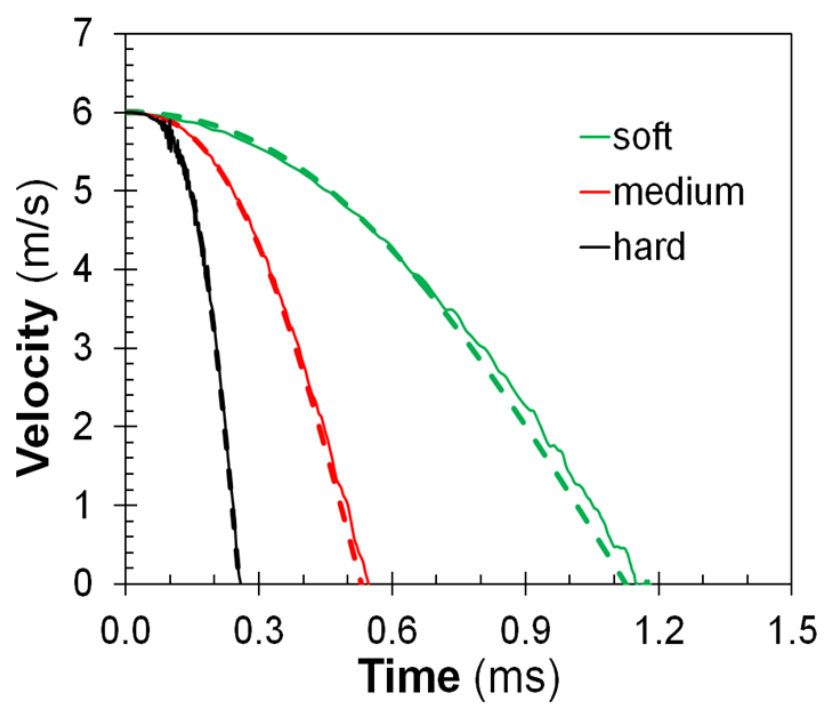

(b)

Fig. 9 Results of the numerical simulations for different substrate stiffnesses: (a) indentation depth vs. time; (b) velocity vs. time. The dashed lines refer to the SDOF spring-mass model whereas the solid lines to the rigorous finite element solution in dynamics. 
1

2

3

4

5

6

7

8

9

10

11

12

13

14

15

16

17

18

19

20

21

22

23

24

25

26

27

28

29

30

31

32

33

34

35

36

37

38

39

40

41

42

43

44

45

46

47

48

49

50

51

52

53

54

55

56

57

58

59

60

61

62

63

64

65

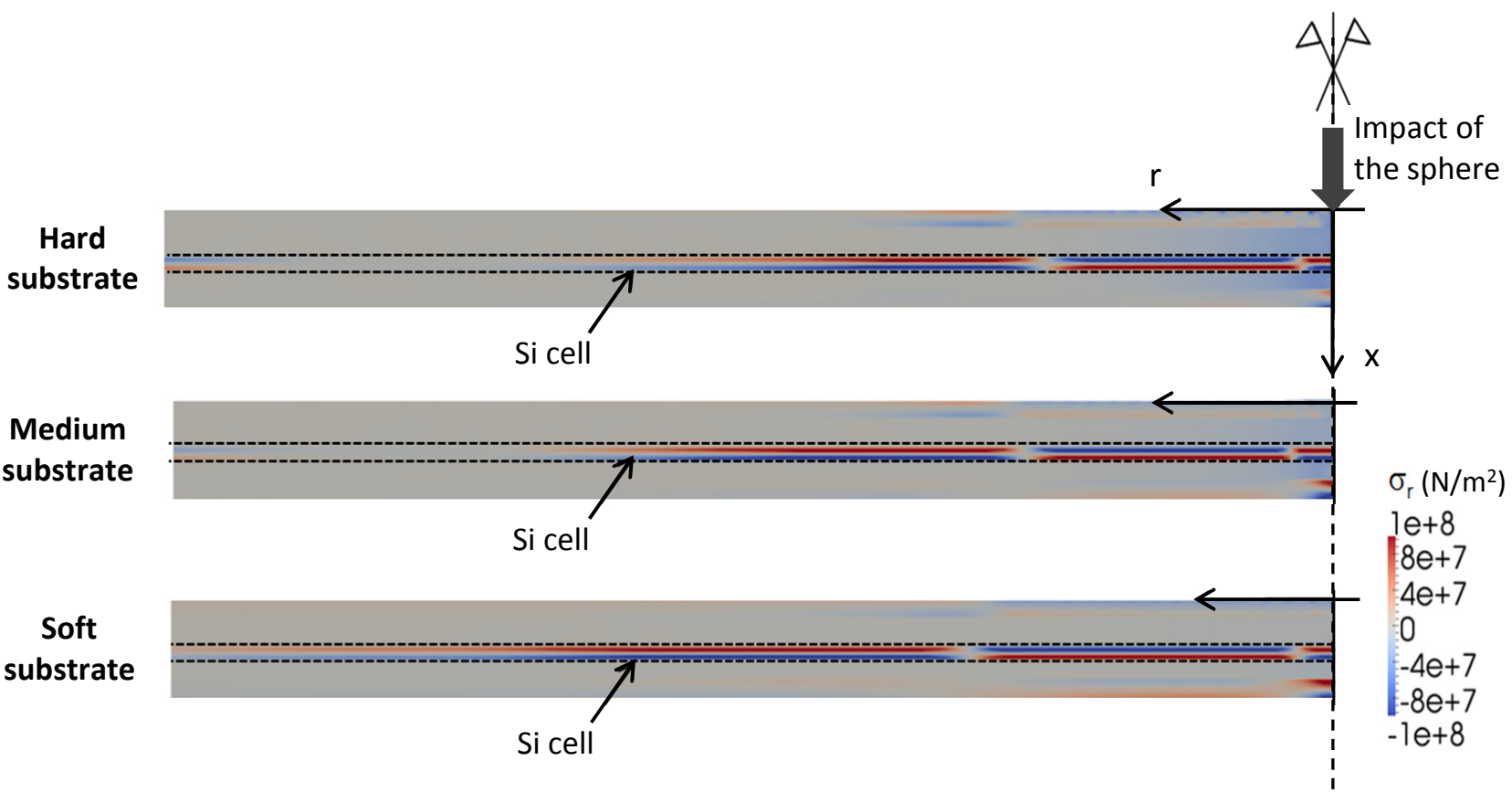

Fig. 10 Contour plot of the radial stress in the PV module for the three considered substrates. These predictions are obtained based on the SDOF method. 
1

2

3

4

5

6

7

8

9

10

11

12

13

14

15

16

17

18

19

20

21

22

23

24

25

26

27

28

29

30

31

32

33

34

35

36

37

38

39

40

41

42

43

44

45

46

47

48

49

50

51

52

53

54

55

56

57

58

59

60

61

62

63

64

65

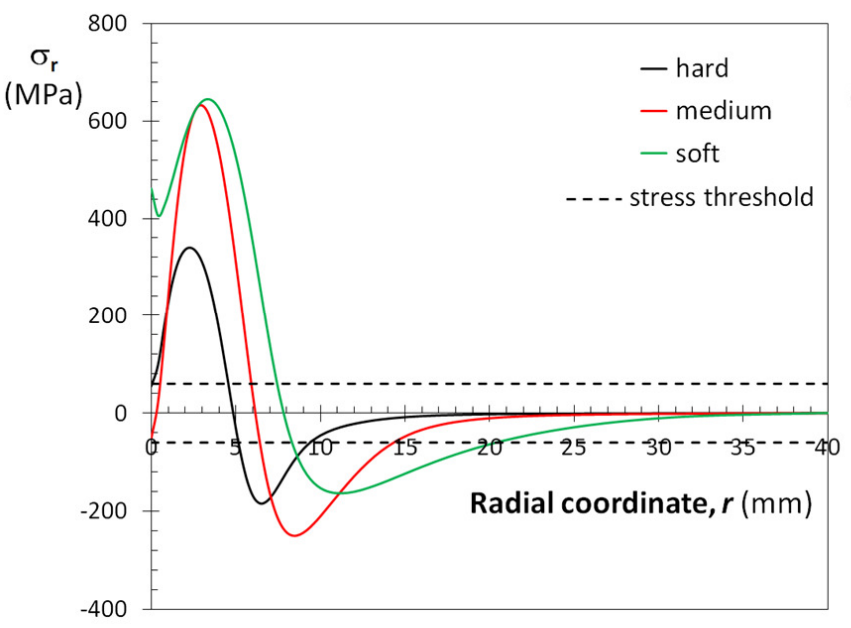

(a)

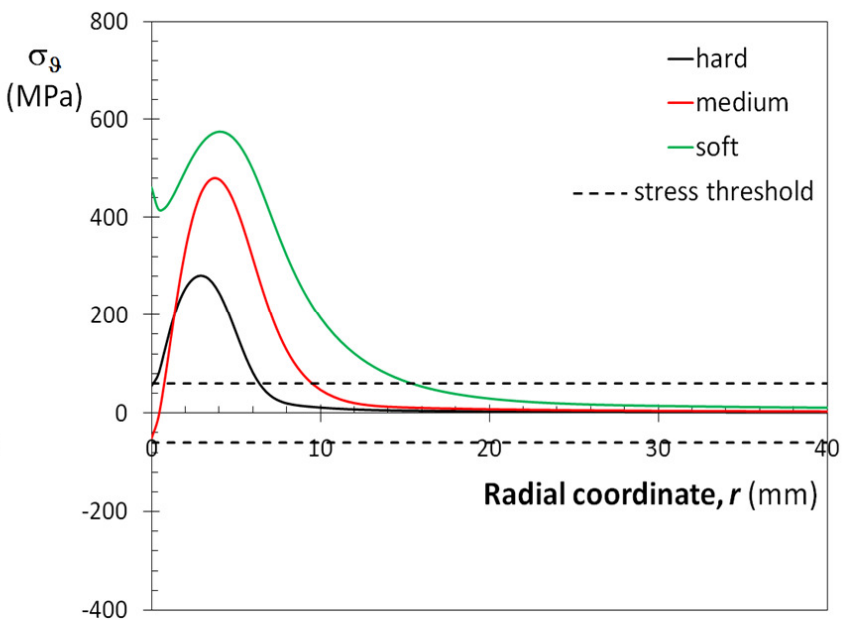

(b)

Fig. 11 Stress distributions along the radial coordinate at the bottom surface of the Si cell predicted by the SDOF method: (a) radial stress; (b) circumferential stress. 
1

2

3

4

5

6

7

8

9

10

11

12

13

14

15

16

17

18

19

20

21

22

23

24

25

26

27

28

29

30

31

32

33

34

35

36

37

38

39

40

41

42

43

44

45

46

47

48

49

50

51

52

53

54

55

56

57

58

59

60

61

62

63

64

65

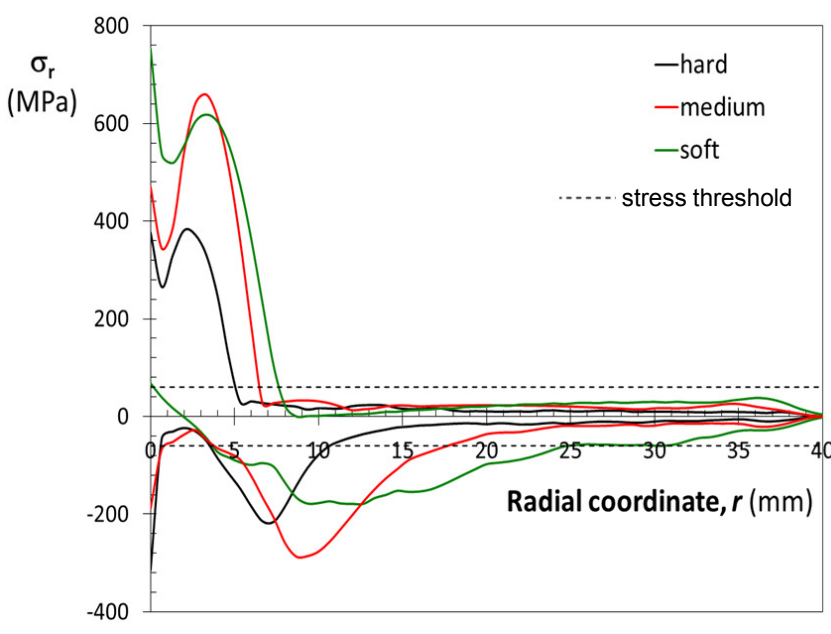

(a)

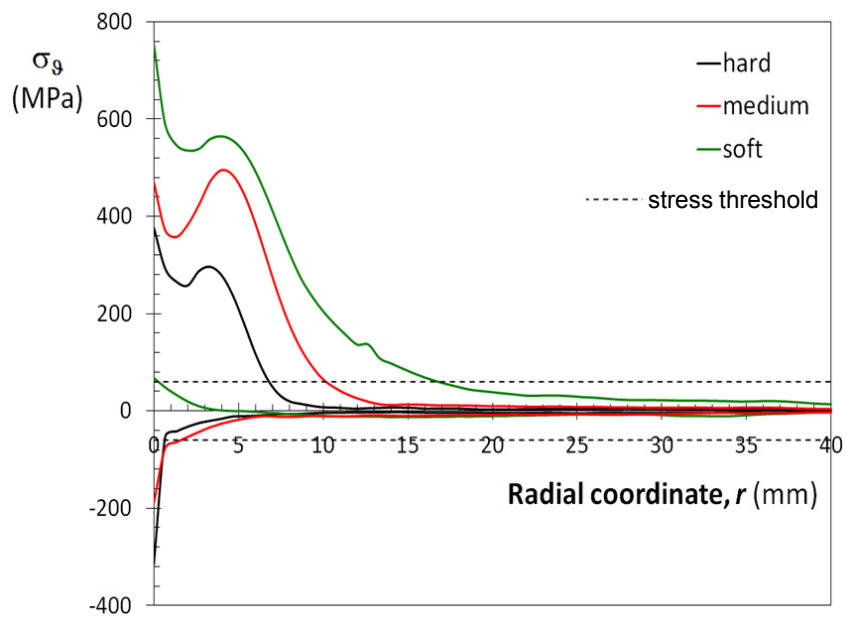

(b)

Fig. 12 Envelopes of the stresses along the radial coordinate at the bottom surface of the Si cell predicted by dynamic finite element simulations: (a) radial stress; (b) circumferential stress. 
1

2

3

4

5

6

7

8

9

10

11

12

13

14

15

16

17

18

19

20

21

22

23

24

25

26

27

28

29

30

31

32

33

34

35

36

37

38

39

40

41

42

43

44

45

46

47

48

49

50

51

52

53

54

55

56

57

58

59

60

61

62

63

64

65

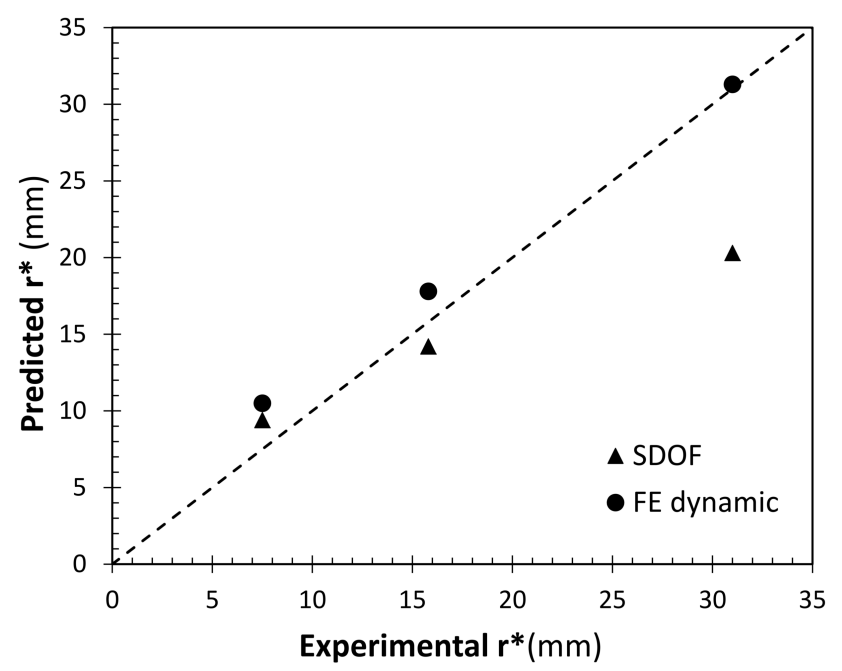

Fig. 13 Comparison between experimental results and numerical predictions of the extension of the silicon area cracked by circumferential cracks, $r^{*}$. 


\section{List of Tables}

1 Material parameters used in the finite element simulations. . . . . . . . . . . . . . . . . . 28 
1

2 3 4 5 5 